1 LI-Seq: A Cost-Effective, Low Input DNA method for Whole Genome Library Preparation

3 Authors: Teia M. Schweizer ${ }^{1^{*}}$, Matthew G. DeSaix ${ }^{1}$, Kristen C. Ruegg ${ }^{1}$

4

$5 \quad *$ Corresponding Author: Teia.Schweizer@gmail.com

$6{ }^{1}$ Department of Biology, Colorado State University, Fort Collins, CO, 80525

\title{
7 Summary
}

8 1. Samples from species of high conservation concern are often low in total genomic DNA. Whole Genome Sequencing (WGS) can provide many insights that can be used to aid in species conservation, but current methods for working with low quality and low input samples can be cost prohibitive for population level genomic analyses. Thus, there is an urgent need for a cost-effective method of preparing WGS libraries from low input DNA samples.

2. To bridge the gap between sampling techniques commonly used in conservation genetics that yield low quality and low input DNA and the powerful tool of WGS, we developed LI-Seq, a more efficient method that successfully produces libraries from low quality DNA with as low input as 0.48 ng of DNA, with an average final library size of 300500 base pairs. quality, low input and high quality, high input starting material using our protocol. We 
conclude that our new method will facilitate high-throughput WGS on low quality, low input samples, thus expanding the power of genomic tools beyond traditional high quality samples.

24 Keywords: whole genome sequencing, low input DNA, low quality DNA, conservation genomics,

25 cost-effective, population genetics

\section{Introduction}

27 The field of genomics began developing in the 1990s with whole genome shotgun sequencing of

28 bacteria (Weissenbach 2016) and has advanced rapidly with the improvement of High-

29 Throughput Sequencing (HTS) techniques (Goodwin et al 2016). The ability to sequence whole

30 genomes with relative ease has opened new research avenues and made it possible to estimate

31 fundamental population genetic parameters with increasing precision in both model and non-

32 model organisms (Allendorf, Hohenloe, and Luikart 2010; Ouborg et al 2010). Of particular

33 relevance to ecologists and conservation biologists, HTS has made it possible to investigate

34 previously challenging topics such as the genetic basis of local adaptation, patterns of

35 inbreeding across the genome, and how species adapt to changing climate conditions (Kohn et

36 al 2006; Ruegg et al 2018; Allendorf, Hohenloe, and Luikart 2010; Ouborg et al 2010). As a

37 result, genomic tools are revolutionizing the fields of ecology, evolution, and conservation

38 biology. 
40 Despite the proliferation of HTS methods for model organisms (Ekblom and Galindo 2011), there

41 remain a number of technical and financial limitations to the widespread use of genomic

42 approaches in situations where the amount of input DNA maybe limited and costs are a

43 concern. While the cost of sequencing has dramatically decreased in the last two decades

44 (Goodwin et al 2016), it is often still prohibitively high for use in population-level studies where

45 hundreds or thousands of individuals must be sequenced (Fuentes-Pardo and Ruzzante 2017).

46 Methods modified from commercially available whole genome sequencing (WGS) library

47 preparation kits offer low coverage options at a fraction of the cost per individual, making them

48 suitable for population genetics studies (Therkildsen and Palumbi 2017; Kryazhimskiy et al 2014;

49 Baym et al 2015). However, these methods still typically require high quality and high input DNA

50 and are not optimized to efficiently amplify smaller target library sizes. Such high quality and

51 quantity DNA can be difficult to attain when working with threatened, endangered, or cryptic

52 species, where ethical and logistical challenges are often prohibitive (Kohn et al 2006; Ouborg et

53 al 2010). However, samples that yield low quality and quantity DNA have previously found

54 limited use in whole genome studies unless potentially cost-prohibitive library preparation kits

55 or methods are employed (Taylor et al 2020). Given the immense potential benefits of analyzing

56 whole genomes for effective wildlife conservation and management efforts (Funk et al 2012;

57 Ryder 2005; Russello et al 2015), there is an urgent need for a cost-effective method of

58 preparing WGS libraries from low quality and quantity DNA samples. 
60 Low quality and quantity samples are often a hallmark of noninvasive or minimally invasive

61 sampling techniques. Noninvasive genetic sampling methods first gained recognition in 1992

62 when DNA was successfully extracted from passively-collected hair for a genetic study of an

63 endangered bear species (Taberlet and Bouvet 1992). Since then, noninvasive genetic sampling

64 has been successfully used in genetic studies across myriad taxonomic groups (Stenglein et al

65 2010; Valiere et al 2003; Roques et al 2014; Regnaut et al 2006). Noninvasive sampling

66 encompasses samples such as saliva, hair, feces, or feathers, collected without capturing,

67 handling, or otherwise disturbing the study organism (Waits et al 2005). Minimally invasive

68 sampling entails capturing or handling a study organism with minimal invasion or tissue

69 collection (e.g. feather pulls and buccal swabs) (Carroll et al 2017). In recent years, noninvasive

70 and minimally invasive sampling methods have gained popularity, especially for use in

71 monitoring threatened and endangered species (Lukacs and Burnham 2005; Fuentes-Pardo and

72 Ruzzante 2017). However, noninvasively or minimally invasively collected samples typically yield

73 lower concentrations of DNA which can limit their use in whole genome studies unless

74 expensive library preparation kits or library preparation methods are employed (Taylor et al

75 2020). Given the immense potential benefits of analyzing whole genomes for effective wildlife

76 conservation and management efforts (Funk et al 2012; Ryder 2005; Russello et al 2015), there

77 is an urgent need for a cost-effective method of preparing WGS libraries from noninvasively or

78 minimally invasively collected samples. 
80 Although low cost methods for WGS exist (Therkildsen and Palumbi 2017; Kryazhimskiy et al

81 2014; Baym et al 2015), they still require a prohibitively large amount of high quality input DNA

$82(2.5 \mathrm{ng})$ for many conservation applications. More specifically, further analysis of these methods

83 reveals that much of the DNA is wasted during the library preparation step due to the fact that

84 the average fragment size produced from these methods is $1 \mathrm{~kb}$, but the average fragment size

85 needed for many common sequencing platforms, such as Illumina, is $300-500$ base pairs. Thus,

86 DNA above 500 base pairs is often removed prior to sequencing. To bridge the gap between

87 sampling techniques commonly used in conservation genetics that yield low quality DNA and

88 the powerful tool of WGS, we developed a more efficient method that successfully produces

89 libraries from low quality DNA with as low input as 0.48 ng of DNA, with an average final library

90 size of $300-500$ base pairs.

91

92 We demonstrate the utility of our method for producing high quality sequencing data at a

93 fraction of the cost of traditional library preparation methods using DNA extracted from a single

94 flight feather calamus, or quill, of a small (8-9 grams) passerine bird, the American Redstart

95 (Setophaga ruticilla). We compare the sequence data from our low input DNA library (from

96 feather) to those generated from a high input library (from blood) and demonstrate that our

97 method produces comparable sequence quality for both low and high input DNA sources. These

98 results have important implications for conservation genomics research seeking to maximize

99 efficient sequencing from low input DNA samples. 
100 Materials and Methods

101 Library Preparation for Sequencing

102 We identified three key parameters in other methods (i.e. Therkildsen and Palumbi 2017) that

103 we could optimize in order to target the ideal fragment distribution and avoid loss of critical

104 DNA when working with low input DNA samples. The three key parameters modified herein

105 were: (1) the ratio of tagmentation transposome (which cleaves DNA and adds an adapter for

106 indices) to input DNA quantity, (2) the duration of tagmentation incubation, and (3) the duration

107 of the indexing PCR elongation time.

108

109 In order to compare sequencing quality from high and low input and quality DNA libraries and

110 assess the efficiency of our method, we extracted DNA from 50 high DNA quantity and quality

111 bird blood samples and 50 low DNA quantity and quality feather calamus tips of the American

112 Redstart. For blood, DNA was extracted from between 50-100 $\mu \mathrm{L}$ of whole blood stored in

113 Queen's Lysis Buffer ( $80 \mu \mathrm{L}$ of whole blood plus $300 \mu \mathrm{L}$ of buffer), using Qiagen DNEasy Blood

114 and Tissue Kit and eluted into $100 \mu \mathrm{L}$ of provided AE buffer. For extractions from feathers, like

115 other low-quality samples, maximizing DNA yield is critical. Therefore, we followed the Qiagen

116 protocol but with the following modifications. To each sample, we added $10 \mu \mathrm{L}$ of $1 \mathrm{M}$

117 Dithiothreitol (DTT) to the initial lysis step to aid in breaking down disulfide bonds found in the

118 keratin of feathers. Flowthrough after the first filtration step when lysate was transferred to the

119 spin column was pipetted back onto the filter for a second centrifugation. Prior to the final

120 elution step, AE buffer was placed in an incubator at $56{ }^{\circ} \mathrm{C}$. During the final elution step, AE 
121 buffer was left to incubate on the filter for five minutes instead of two. We eluted feather

122 extractions into $400 \mu \mathrm{L}$ (two rounds of $200 \mu \mathrm{L}$ elutions through the spin column as

123 recommended by Qiagen protocol for maximum yield). Prior to proceeding with library prep, we

124 concentrated feather DNA extractions using a 1:1 ratio of Serapure beads (Faircloth and Glenn

125 2014) from $400 \mu \mathrm{L}$ to $15 \mu \mathrm{L}$ and eluted into 10mM Tris-Hcl (Figure 1, step 1a).

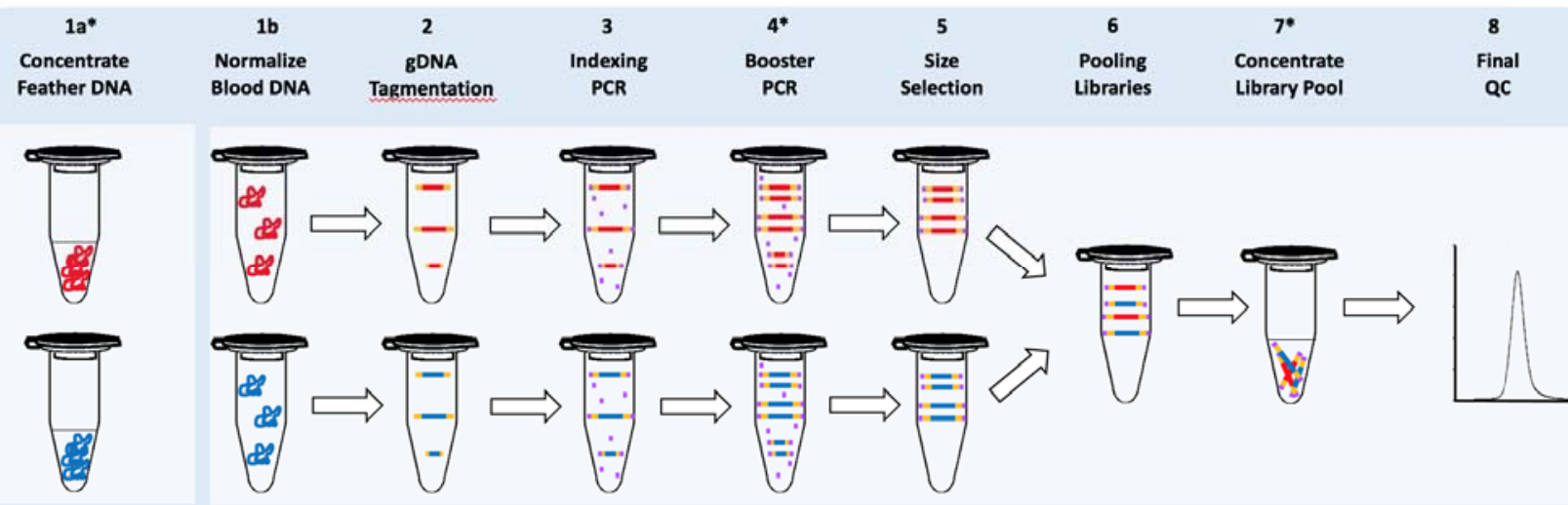

127 Figure 1. Lab workflow diagram for LI-Seq method. Steps 1 through 8 follow full protocol (see 128 Supplementals). Steps with * after number denote steps where modifications to typical WGS 129 library preparation methods were implemented. Low input samples start with step 1a and high 130 input samples start with step $1 b$.

132 To ensure that final library concentrations will be similar across samples, we quantified each

133 DNA extraction using a Qubit dsDNA High Sensitivity Assay Kit (Invitrogen) and normalized each

134 sample to a concentration of $0.48 \mathrm{ng} / \mu \mathrm{L}-4.5 \mathrm{ng} / \mu \mathrm{L}$, with a target of $2.5 \mathrm{ng} / \mu \mathrm{L}$ (Figure 1, step 1a

135 and $1 \mathrm{~b})$. To fragment the DNA and "tag" it with Nextera adapters, we added $2.50 \mu \mathrm{L}$ of TD

136 Buffer and $0.5 \mu \mathrm{L}$ of TDE1 Enzyme (Illumina) to $1 \mu \mathrm{L}$ of normalized DNA and incubated the

137 samples in a thermocycler at $55^{\circ} \mathrm{C}$ for 20 minutes (Figure 1, step 2). 
139 To amplify the tagmented DNA and add Nextera indexing adapters for sequencing, we pipetted

$1401 \mu \mathrm{L}$ of each index primer into the appropriate well of tagmented DNA until all samples had a

141 unique pair of dual indexes. We then added 6.0 $\mu \mathrm{L}$ of Kapa Hifi Hotstart Mix (KMM; Kapa

142 Biosystems) before running in a thermocycler as follows: held at $72^{\circ}$ for 3 minutes, held at $98^{\circ}$

143 for 2 minutes and 45 seconds, cycled 8 times through $98^{\circ}$ for 15 seconds, then $62^{\circ}$ for 30

144 seconds, then $72^{\circ}$ for 30 seconds, held at $72^{\circ}$ for 1 minute, and then held at $4^{\circ}$ until removed

145 from thermocycler (Figure 1, step 3). As per the Therkildsen and Palmubi (2017) method, this

146 indexing PCR had more cycles than the original Illumina protocol and was broken into two

147 stages (Indexing PCR and "Reconditioning PCR, " which was renamed Booster PCR in this

148 method). Additional cycles were added in the Therkildsen and Palumbi (2017) method because

149 the tagmented DNA was not purified prior to Indexing PCR, making the PCR reaction less

150 efficient. To further amplify, or "boost," copies of indexed DNA without using additional Nextera

151 indices, we added 7.6 $\mu \mathrm{L}$ of KMM, $4.4 \mu \mathrm{L}$ of ultrapure water, and $1.6 \mu \mathrm{L}$ each of a custom 10uM

152 primer pair (P1=AATGATACGGCGACCACCGA; P2=CAAGCAGAAGACGGCATACGA) to each library.

153 We ran the samples in a thermocycler as follows: held $95^{\circ}$ for 5 minutes, cycle 4 times through

$15498^{\circ}$ for 20 seconds, then $62^{\circ}$ for 20 seconds, then $72^{\circ}$ for 2 minutes, hold at $72^{\circ}$ for 2 minutes,

155 and then held at $4^{\circ}$ until removed from the thermocycler (Figure 1, step 4).

157 To purify the PCR product and remove undesirable fragments, we followed standard Ampure

158 bead protocol (Beckman Coulter) using a 0.7:1 bead to DNA ratio which will remove below

159 approximately $320 \mathrm{bp}$ and eluted into $30 \mu \mathrm{L}$ of $10 \mathrm{mM}$ Tris-Hcl (Figure 1, step 5). In order to 
160 avoid overrepresentation of one individual during whole genome resequencing, we then

161 quantified using a Qubit dsDNA High Sensitivity Assay Kit (Invitrogen) and pooled an equal

162 number of copies of each sample into a $1.5 \mathrm{~mL}$ tube (Figure 1, step 6). Finally, in order to

163 increase the final concentration of the pooled libraries and increase sequencing efficiency, we

164 then followed the standard Ampure double-sided size selection protocol, using a 0.63:1 bead to

165 DNA ratio to remove large fragments and a 0.73:1 bead to DNA ratio to remove small

166 fragments, and eluted into $30 \mu \mathrm{L}$ of $10 \mathrm{mM}$ Tris- $\mathrm{HCl}$ (Figure 1, step 7). After the pooled library

167 has been concentrated and double size selected (either with or without the optional

168 reconditioning PCR), we perform final quality control (QC) with Qubit quantification and

169 Tapestation 2200 fragment distribution analysis (Agilent) (Figure 1, step 8).

171 To address issues of overamplification, also called 'PCR bubble,' we encountered while using

172 Therkildsen and Palumbi's original method (2017) with our low input DNA, we added an

173 optional 'reconditioning PCR' step which provides additional reagents, especially primers, so

174 that the PCR product does not anneal to itself (Thompson et al 2002). To recondition a final

175 pooled library with overamplification, we added $12 \mu \mathrm{L}$ of pooled, size selected library and added

$1767.6 \mu \mathrm{L}$ of KMM, $1.6 \mu \mathrm{L}$ of 10uM P1 (AATGATACGGCGACCACCGA), $1.6 \mu \mathrm{L}$ of 10uM P2

177 (CAAGCAGAAGACGGCATACGA), and $4.4 \mu \mathrm{L}$ of ultrapure water. We then ran it in a thermocycler

178 as follows: held $95^{\circ}$ for 5 minutes, cycled once through $98^{\circ}$ for 20 seconds, then $62^{\circ}$ for 20

179 seconds, then $72^{\circ}$ for 2 minutes, held at $72^{\circ}$ for 2 minutes, and then held at $4^{\circ}$ until removed

180 from the thermocycler. Next, we used Ampure beads to clean up and performed an additional 
181 double size selection and bead cleanup before quantifying and running the library through a

182 Tapestation 2200 fragment distribution analyzer (Agilent) for final quality control. This

183 reconditioning PCR is optional and may not be required for all library preparations.

185 Using the above method, we prepared two WGS libraries from American Redstart (Setophaga

186 ruticilla) samples for low (2x) coverage sequencing. One library was prepared with 50 unique

187 blood samples of normalized DNA concentrations between 1.18 - $4.78 \mathrm{ng} / \mathrm{uL}$. The other library

188 was prepared with 50 unique feather samples, extracted from a single feather calamus, with

189 starting DNA concentrations of $0.48-5.7 \mathrm{ng} / \mu \mathrm{L}$. Both libraries had one cycle of reconditioning

190 PCR performed on the final library. Libraries were each sequenced on one full $2 \times 150$ bp PE

191 (paired end) HiSeq 4000 lane (Illumina).

192

193 Bioinformatic Analysis and Quality Checking

195 We trimmed the sequence data to remove potential PCR artifacts using the program FastUniq

196 version 0.11.9 (Xu et al 2012). PCR duplicates need to be removed in order to ensure high-

197 quality sequence data in downstream processes such as creating scaffolds in whole-genome

198 sequencing. We mapped reads to an assembled genome of the yellow warbler (Setophaga

199 petechia; Bay et al 2018), using the Burrows-Wheeler Aligner software version 0.7.17 (Li and

200 Durbin 2010). The resulting SAM files were sorted, converted to BAM files, and then indexed

201 using samtools version 1.9 (Li et al 2009). Depth of sequencing coverage at every read position 
202 was calculated using the depth function in samtools (Li et al 2009). The quality of the BAM files

203 for the two different libraries was assessed by comparing the average read depth by individual

204 as well as the average read depth by scaffold. We quantified the GC content of 100 base pair

205 windows in the BAM files from the two libraries using CollectGcBiasMetrics function in Picard

206 version 2.23.1 (Broad Institute 2019). We determined the proportion of reads that passed

207 quality filters for the two libraries using CollectWgsMetrics in Picard (Broad Institute 2019).

208 Two-tailed t-tests were used to compare the quality diagnostics for the different libraries and

209 were implemented in $\mathrm{R}$ version 3.6.2 (R Core Team 2019).

210 Results

211 Library Preparation for Sequencing

212 A comparison between libraries prepared using the method of Therkildsen and Palumbi (2017)

213 and our modified method revealed that doubling the ratio of tagmentation enzyme to DNA,

214 increasing the tagmentation time to 20 minutes, and decreasing the indexing PCR elongation

215 time to 30 seconds resulted in maximization of fragments in the target distribution (Table 1;

216 Figure 2).

217

218 Table 1. Matrix of the original (Therkildsen) protocol parameters and final LI-Seq parameters, 219 tested with 3 high input samples (blood).

\begin{tabular}{|c|c|c|c|c|c|c|c|c|c|}
\hline $\begin{array}{c}\text { Test } \\
\text { Name }\end{array}$ & $\begin{array}{c}\text { total } \\
\text { DNA } \\
\text { (ng) }\end{array}$ & $\begin{array}{c}\text { enzyme } \\
\text { (ul) }\end{array}$ & $\begin{array}{c}\text { buffer } \\
\text { (ul) }\end{array}$ & $\begin{array}{c}\text { total } \\
\text { rxn } \\
\text { vol } \\
\text { (ul) }\end{array}$ & $\begin{array}{c}\text { tagmentatio } \\
\text { n time } \\
\text { (min) }\end{array}$ & $\begin{array}{c}\text { Index } \\
\text { Elongatio } \\
\text { n Time } \\
\text { (sec) }\end{array}$ & $\begin{array}{c}\text { Sample } \\
\text { Number }\end{array}$ & $\begin{array}{c}\text { Final } \\
\text { Conc } \\
\text { (ng/ul) }\end{array}$ & $\begin{array}{c}\text { Avg } \\
\text { fragmen } \\
\text { t length } \\
\text { (bp) }\end{array}$ \\
\hline Original & 2.5 & 0.25 & 1.25 & 2.5 & 5 & 180 & $\mathrm{~A}$ & 8 & 663 \\
\hline
\end{tabular}




\begin{tabular}{|c|c|c|c|c|c|c|c|c|c|}
\hline & & & & & & & B & 8.54 & 438 \\
\hline & & & & & & & C & 8.3 & 439 \\
\hline \multirow{3}{*}{ LI-Seq } & \multirow{3}{*}{2.5} & \multirow{3}{*}{0.5} & \multirow{3}{*}{2.5} & \multirow{3}{*}{4} & \multirow{3}{*}{20} & \multirow{3}{*}{30} & $A$ & 2.79 & 386 \\
\hline & & & & & & & B & 2.69 & 309 \\
\hline & & & & & & & C & 2.39 & 397 \\
\hline
\end{tabular}

Figure 2. Tapestation 2200 gel of Therkildsen protocol conditions and final LI-Seq conditions from Table 1. Yellow bands show preferred fragment range (320-500bp) for HiSeq 4000 as

223 recommended by Novogene. Individuals A, B and C were duplicated between the Therkildsen 224 Blood and LI-Seq Blood, with the conditions described in Table 1. Individual D was feather DNA 225 prepared using only the LI-Seq method.

227 Using these modified methods, we successfully prepared two libraries from American Redstart

228 DNA. The library from 50 high input samples (blood) had a final concentration of $12.1 \mathrm{ng} / \mu \mathrm{L}$, a

229 molarity of $39.3 \mathrm{nM}$, and an average library size of $466 \mathrm{bp}$ (Figure 3d). The library from 50 low

230 input samples (feathers) had a final concentration of $2.56 \mathrm{ng} / \mu \mathrm{L}$, a molarity of $8.56 \mathrm{nM}$, and an

231 average library size of 453 bp (Figure 3f). 


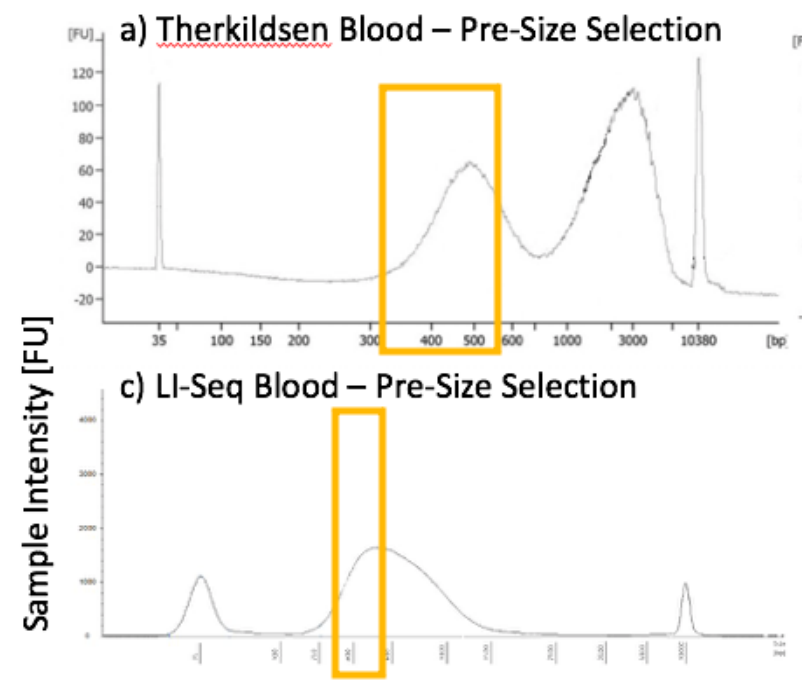

e) LI-Seq Feather - Pre-Size Selection b) Therkildsen Blood - Post-Size Selection
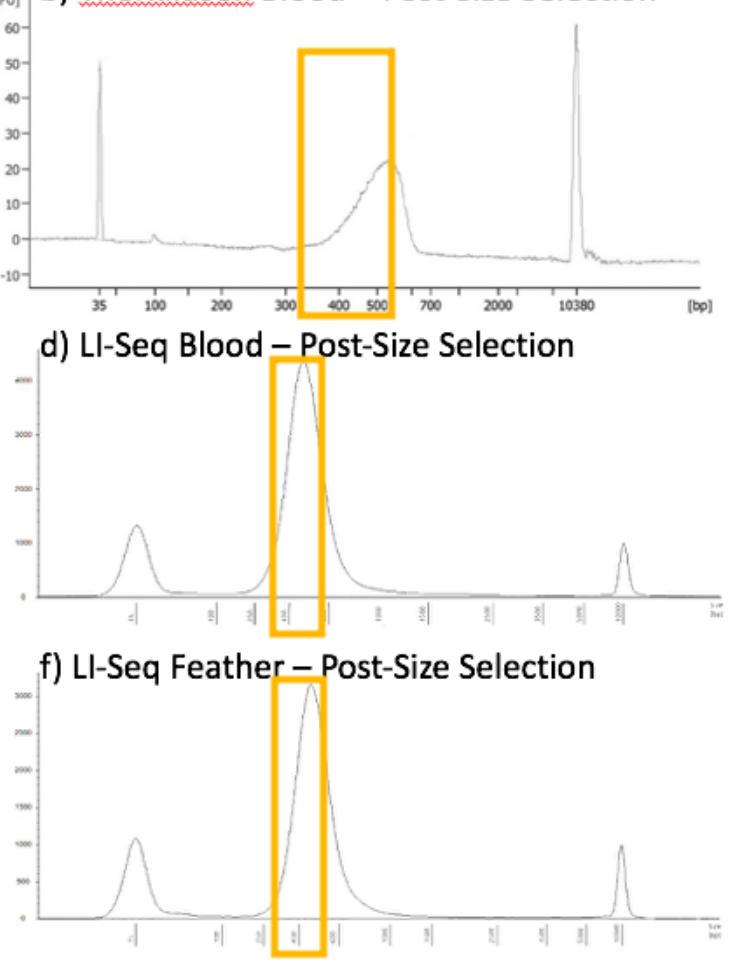

Fragment Length [basepairs]

233 Figure 3. Final library fragment analysis of a library prepared using the original Therkildsen and 234 Palumbi protocol (2017), before (a) and after (b) size selection, run on a Bioanalyzer (Agilent). 235 The second peak of (a) indicated overamplification. The final libraries of the high input (blood) 236 and low input (feather) samples were run on a Tapestation 2200 (Agilent) prior to size selection 237 (high input (c) and low input (e)) and after size selection (high input (d), low input (f)). The x-axis 238 is the size of the DNA fragment in basepairs, and the y-axis is the sample intensity which is 239 correlated with sample concentration. Note the different $x$ - and $y$-axis scales. Yellow boxes 240 indicate the desired fragment size of $\sim 320-500$ bps.

242 Bioinformatic Analysis and Quality Checking

243 The depths of coverage of the sequence data from the two libraries were not significantly 244 different $(t=1.06, d f=98, p$-value $=0.29)$, but the high input library had a slightly higher depth 245 (mean 1.79, standard deviation 0.52; Figure 3a) than the low input library (mean 1.70, standard 246 deviation 0.38; Figure 3a). The high input library had individuals that had a slightly higher 
247 proportion of the genome with sequence data (mean 0.66, standard deviation 0.06; Figure 3b)

248 than the low input library (mean 0.64, standard deviation 0.08; Figure 3b), but this difference

249 was not statistically significant $(t=1.85, \mathrm{df}=98, \mathrm{p}$-value $=0.07)$. The difference in $\mathrm{GC}$

250 distribution between the two libraries was non-significant $(42.9 \%$; $t=1.94 e-14, d f=100, p$-value

$251=1$ ), with the mean GC content of the high input library being $42.7 \%$ and $42.9 \%$ for the low

252 input library (Figure 4C). The sequence data from these two libraries also had very similar

253 patterns of coverage across the scaffolds in the genome (Figure 5). The similarity in coverage

254 across scaffolds shows that certain genomic regions are not being over- or under-amplified in

255 either of the libraries due to a difference in quality and quantity of input DNA. All BAM quality

256 metrics for the two libraries produced comparable results (Table 2).

a

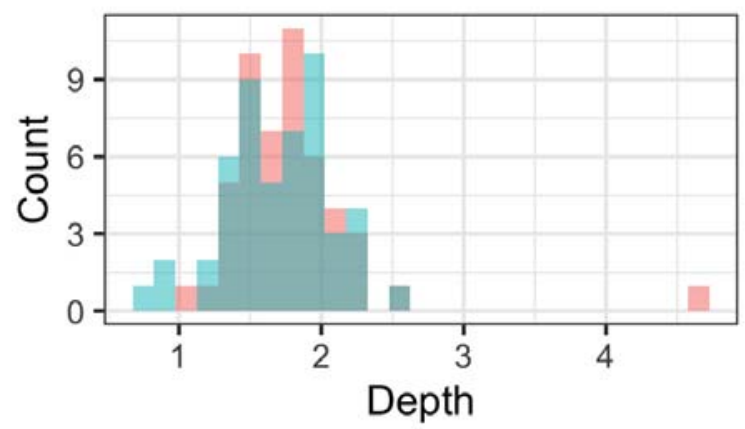

b

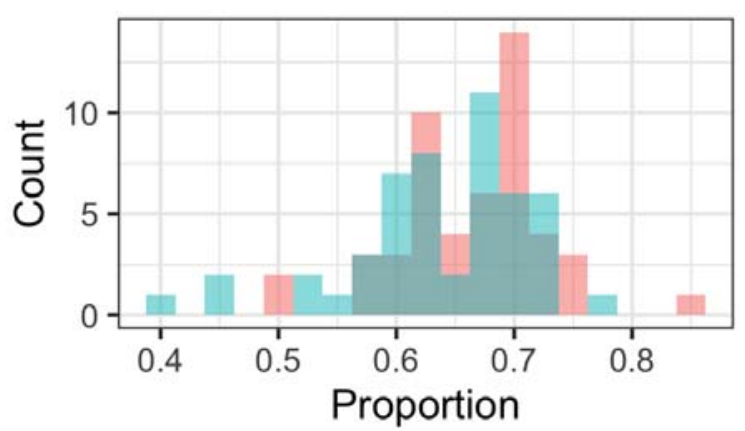

C

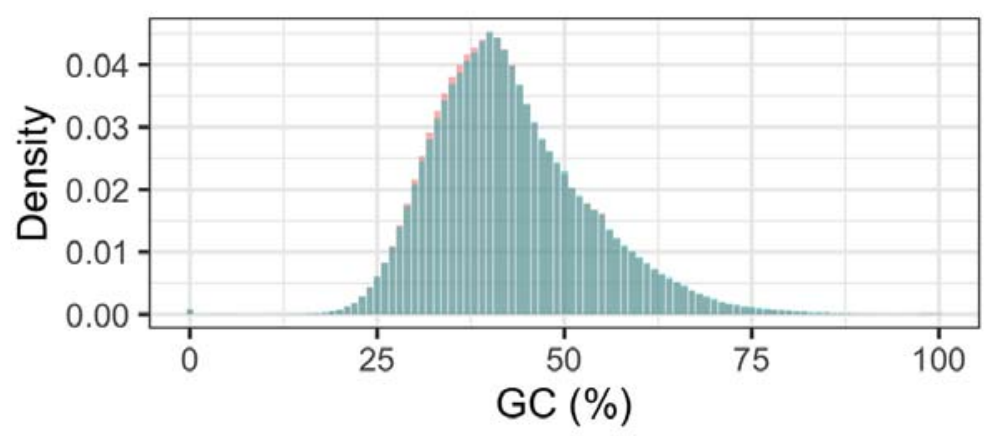

Plate

High-input library (Blood)

Low-input library (Feathers) 
Figure 4. Both histograms of (a) average depth across the genome by individual and (b) proportion of genome with sequence data by individual overlapped considerably between the two libraries and neither measures were significantly different (depth: $t=1.06, d f=98, p$-value $=0.29$; proportion: $\mathrm{t}=1.85, \mathrm{df}=98, \mathrm{p}$-value $=0.07)$. $\mathrm{GC}$ content was determined for 100 base pair region windows in each individual and then averaged across individuals for each of the two plates. (c) The bins of GC content (ranging from 0 to 100\%) were nearly identical for the two libraries $(t=1.94 \mathrm{e}-14, \mathrm{df}=100, \mathrm{p}$-value $=1)$. Data from the high input library from blood samples are shown in red, the low input feather library in light blue, and the overlapping data in

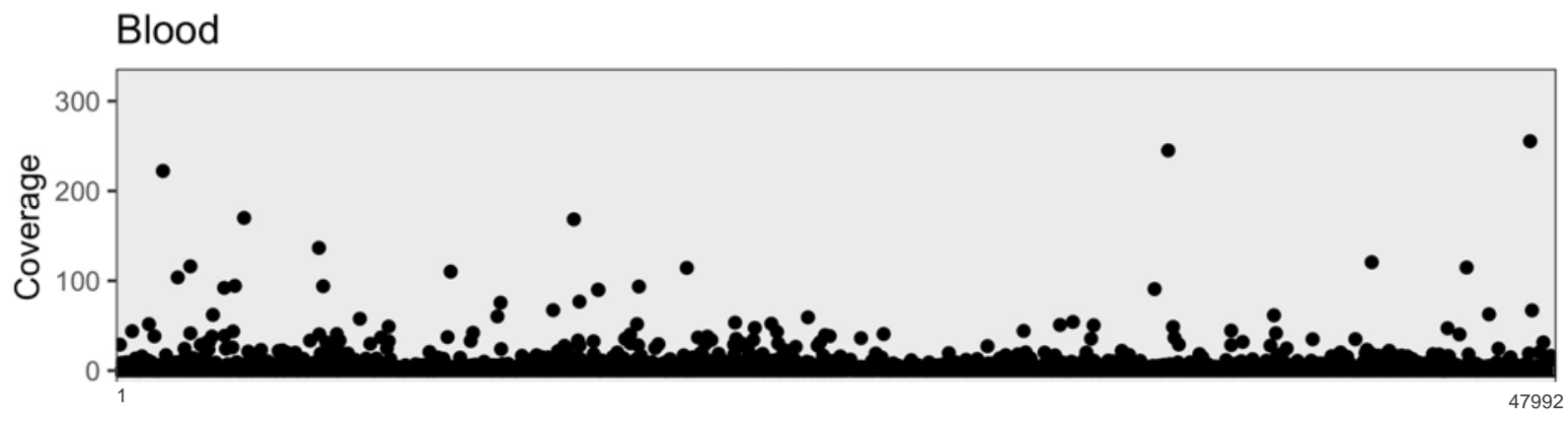

Scaffold

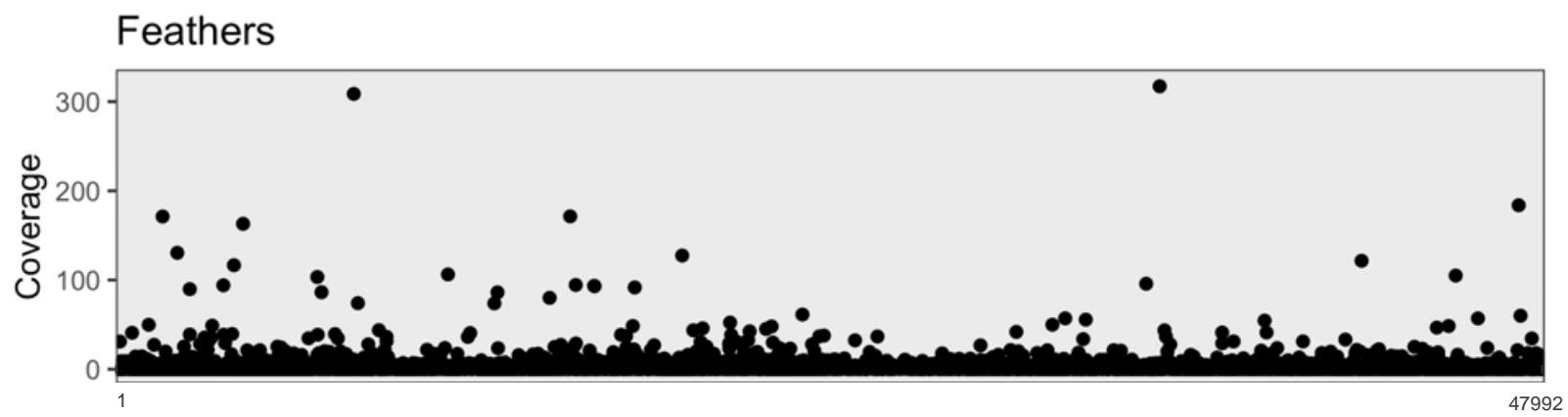

Scaffold

270 Figure 5. Mean coverage by scaffold averaged across all individuals for the two libraries reveal similar patterns in coverage across the genome between the two libraries.

Table 2. BAM quality statistics: the average proportion of reads with adaptors (Adaptors), mapping quality less than 20 (MapQ), marked as duplicates (Duplicate), without a mapped mate pair (Unpaired), quality score less than 20 (BaseQ), and at least $1 x(1 x)$ and $5 x(5 x)$ coverage after removing low quality reads.

\begin{tabular}{|l|l|l|l|l|l|l|l|}
\hline Library & Adaptors & MapQ & Duplicate & Unpaired & BaseQ & $1 \mathrm{x}$ & $5 \mathrm{x}$ \\
\hline
\end{tabular}




\begin{tabular}{|l|l|l|l|l|l|l|l|}
\hline $\begin{array}{l}\text { High input } \\
\text { (Blood) }\end{array}$ & 0 & 0.110 & 0 & 0.001 & 0.017 & 0.685 & 0.049 \\
\hline Low input & & 0.117 & 0 & 0.001 & 0.015 & 0.657 & 0.039 \\
\hline
\end{tabular}

\section{Discussion}

278 In conservation genetics, researchers must often work from low quality and low input DNA

279 samples, especially in the case of noninvasive or minimally invasive sampling. These types of

280 samples can be a challenge to use with many genomic techniques, such as WGS, which typically

281 require high input and quality input DNA. Yet, collecting high input samples (like blood and

282 tissue) can present ethical, logistical, and financial roadblocks.

283

284 The potential knowledge gained from utilizing WGS and similar genomic tools can provide

285 insights not yet achievable from other methods. WGS can be a powerful tool for management in

286 conservation efforts but, until now, has been challenging and or cost-prohibitive when working

287 with low input, low quality DNA. Here we have described LI-Seq, a method for cost-effective

288 WGS library preparation that can be used for both high quality, high input and low quality, low

289 input samples. Results suggest that, with the modifications described above, one can

290 successfully produce high quality sequence data from DNA with input as low as $0.48 \mathrm{ng}$, for a

291 fraction of the cost of traditional library preparation methods. More specifically with this

292 method, we were able to prepare approximately 12 libraries for the same price as a single 
293 library with a more traditional WGS library preparation kit, thus allowing us to sequence more

294 than an order of magnitude more samples. Overall, the increase in efficiency and cost-

295 effectiveness provided by our method will allow conservation biologists to more broadly apply

296 WGS methods to samples collected with non-invasive or minimally invasive methods.

Another potential challenge of using low input DNA with WGS methods is biased or incomplete

amplification of the genome due to the low number of copies of the entire genome present

300 (Meynert et al 2014). To assess this, we prepared a library from high quality, high input DNA

301 (extracted from blood) in addition to preparing a library from low quality, low input DNA

302 (extracted from feathers) using the same protocol. The final library molarity and fragment

303 distribution was similar with both DNA sources, suggesting that the lower input DNA yielded

304 equally high-quality libraries as high input DNA. In addition to impacting the quality of the final

305 library, low input DNA could result in biased amplification leading to preferential sequencing of

306 certain regions of the genome and result in less equal coverage across the genome than with

307 high input DNA. The observed correspondence in patterns of genome-wide coverage between

308 the libraries is to be expected in the absence of external influences causing biases related to the

309 quality of input DNA (Ekblom, Smeds, and Ellegren 2014). Additionally, we checked for

310 differential GC bias between the two libraries because it can indicate that biases were

311 introduced during library preparation (Sims et al 2014). Our breadth of genome coverage (i.e.

312 proportion of the genome sequenced) of $64 \%$ and $66 \%$ for the two libraries (high input and low

313 input respectively) is comparable to that of mammalian genomes sequenced at similar depth 
314 (Green 2007). This breadth of coverage is suitable for numerous conservation genomic

315 applications (e.g. identifying inbreeding across the genome) and resource efficient considering

316 that approximately 30x coverage depth is required to achieve 95\% coverage breadth (Sims et al

317 2014). Overall, our results suggest that when our method is employed, libraries prepared from

318 low input and high input DNA both produce high quality sequence data.

320 When applying this protocol to other species and sample types, researchers may want to think

321 about a couple important considerations. First, the genome size of the study organism should

322 be considered. The protocol presented herein is an excellent option for use with organisms with

323 a small genome, as the protocol was optimized using DNA from birds which on average have a

324 genome size of $1.1 \mathrm{~Gb}$. For species with larger genomes, more sequencing is required to achieve

325 the same level of coverage, and therefore may require optimized methods and will have a

326 relatively higher cost as well. Additionally, the ratio of tagmentation enzyme to input DNA may

327 need to be adjusted in order to maintain a similar average fragment size. The tagmentation

328 enzyme is one of the most expensive components of this method, so increasing the amount of

329 enzyme per sample could also increase the per sample cost. Second, when considering

330 combining libraries from low quality, low input and high quality, high input samples into a single

331 sequencing run, researchers may want to take additional steps to ensure equal representation

332 of the libraries. Specifically, performing a double size selection on the individual libraries prior

333 to quantification and pooling can help ensure that the most accurate concentrations are used

334 when pooling individuals for sequencing and, therefore, the most equal sequencing effort per 
335 sample will occur (Zamudio et al unpublished). Overall, with the aforementioned modifications

336 taken into account, the method presented here could be applied more broadly to increase the

337 efficiency and cost-effectiveness of WGS across a multitude of taxa.

338

339 Here we present a cost-effective method for producing WGS libraries using low input DNA from

340 minimally-invasively collected samples. LI-seq provides a much-needed tool to bridge the gap

341 between the conservation management applications of WGS data and frequently collected

342 sample types, such as feathers and other non-invasively collected samples. Although a recent

343 method was published that also provided a method for preparing non-invasively collected

344 samples for WGS (Taylor et al 2020), the per sample cost may be prohibitive for use with

345 population-scale studies for conservation efforts. By providing an efficient, cost-effective WGS

346 method for low quantity and quality DNA samples, we hope conservation management efforts

347 will be able to better take advantage of the applications WGS can provide for enhancing

348 management efforts. 


\section{Acknowledgements}

350 Thank you to Dr. Nina Therkildsen fo sharing her WGS method, Harmony B. Borchardt-Wier for

351 early guidance on trouble-shooting Dr. Therkildsen's protocol, and Dr. Cristian Gruppi for his

352 technical advising. We thank the UC Davis Genome Center for their help with the sequencing.

353 This work was made possible by an NSF CAREER award (008933-00002), an NSF grant Rules of

354 Life grant (007604-00002), and a National Geographic grant (WW-202R-170) to K. Ruegg as well

355 as the Extreme Science and Engineering Discovery Environment (XSEDE) computer cluster,

356 which is supported by National Science Foundation grant ACI-1548562.

\section{References}

Allendorf, F. W., Hohenlohe, P. A., \& Luikart, G. (2010). Genomics and the future of conservation genetics. Nature reviews genetics, 11(10), 697-709.

362 Baym, M., Kryazhimskiy, S., Lieberman, T. D., Chung, H., Desai, M. M., \& Kishony, R. (2015). Inexpensive multiplexed library preparation for megabase-sized genomes. PloS one, 10(5), e0128036. Science, 359(6371), 83-86. Genetic and genomic monitoring with minimally invasive sampling methods. Evolutionary applications, 11(7), pp.1094-1119. ecology of non-model organisms. Heredity, 107(1), 1-15.

378 ultra-deep sequencing of vertebrate mitochondria. BMC genomics, 15(1), 467. 

Serapure). doi: 10.6079. J9MW2F26.

Fuentes-Pardo, A. P., \& Ruzzante, D. E. (2017). Whole-genome sequencing approaches for conservation biology: Advantages, limitations and practical recommendations. Molecular ecology, 26(20), 5369-5406.

386

Funk, W. C., McKay, J. K., Hohenlohe, P. A., \& Allendorf, F. W. (2012). Harnessing genomics for delineating conservation units. Trends in ecology \& evolution, 27(9), 489-496.

389

Goodwin, S., McPherson, J. D., \& McCombie, W. R. (2016). Coming of age: ten years of nextgeneration sequencing technologies. Nature Reviews Genetics, 17(6), 333.

Green, P. (2007). 2× genomes-Does depth matter?. Genome research, 17(11), 1547-1549.

Kohn, M. H., Murphy, W. J., Ostrander, E. A., \& Wayne, R. K. (2006). Genomics and conservation genetics. Trends in ecology \& evolution, 21(11), 629-637.

Kryazhimskiy, S., Rice, D. P., Jerison, E. R., \& Desai, M. M. (2014). Global epistasis makes adaptation predictable despite sequence-level stochasticity. Science, 344(6191), 1519-1522.

Li, H., \& Durbin, R. (2010). Fast and accurate long-read alignment with Burrows-Wheeler transform. Bioinformatics, 26(5), 589-595.

Li, H., Handsaker, B., Wysoker, A., Fennell, T., Ruan, J., Homer, N., ... \& Durbin, R. (2009). The sequence alignment/map format and SAMtools. Bioinformatics, 25(16), 2078-2079.

Lukacs, P. M., \& Burnham, K. P. (2005). Review of capture-recapture methods applicable to noninvasive genetic sampling. Molecular ecology, 14(13), 3909-3919.

Meynert, A.M., Ansari, M., FitzPatrick, D.R., \& Taylor, M.S. (2014). Variant detection sensitivity and biases in whole genome and exome sequencing. BMC bioinformatics, 15(1), pp.1-11.

418

419

Ouborg, N. J., Pertoldi, C., Loeschcke, V., Bijlsma, R. K., \& Hedrick, P. W. (2010). Conservation genetics in transition to conservation genomics. Trends in genetics, 26(4), 177-187.

R Core Team (2019). R: A language and environment for statistical computing. $R$ Foundation for Statistical Computing, Vienna, Austria. URL https://www.R-project.org/. 
Regnaut, S., Lucas, F. S., \& Fumagalli, L. (2006). DNA degradation in avian faecal samples and feasibility of non-invasive genetic studies of threatened capercaillie populations. Conservation Genetics, 7(3), 449-453.

423

Roques, S., Furtado, M., Jácomo, A. T., Silveira, L., Sollmann, R., Torres, N. M., ... \& Palomares, F. (2014). Monitoring jaguar populations Panthera onca with non-invasive genetics: a pilot study in Brazilian ecosystems. Oryx, 48(3), 361-369. (2018). Ecological genomics predicts climate vulnerability in an endangered southwestern songbird. Ecology letters, 21(7), 1085-1096. practice: pairing non-invasive sampling with genomics in conservation. PeerJ, 3, e1106.

Ryder, O. A. (2005). Conservation genomics: applying whole genome studies to species conservation efforts. Cytogenetic and genome research, 108(1-3), 6-15. genetic sampling for monitoring reintroduced wolves. The Journal of Wildlife Management, 74(5), 1050-1058.

Taberlet, P., \& Bouvet, J. (1992). Bear conservation genetics. Nature, 358(6383), 197-197. invasively collected samples. Molecular ecology resources, https://doi.org/10.22541/au.158809437.78730399 hundreds of individually barcoded samples for population and evolutionary genomics in nonmodel species. Molecular ecology resources, 17(2), 194-208. amplifications: formation, consequence and elimination by 'reconditioning PCR'. Nucleic Acids Research, 30(9), 2083-2088. sampling over a period of 10 years. Animal Conservation, 6(1), 83-92. 
463 Waits, L. P., \& Paetkau, D. (2005). Noninvasive genetic sampling tools for wildlife biologists: a

464 review of applications and recommendations for accurate data collection. The Journal of Wildlife

465 Management, 69(4), 1419-1433.

466

467

Weissenbach, J. (2016). The rise of genomics. Comptes rendus biologies, 339(7-8), 231-239.

468

469 Xu, H., Luo, X., Qian, J., Pang, X., Song, J., Qian, G., ... \& Chen, S. (2012). FastUniq: a fast de novo

470 duplicates removal tool for paired short reads. PloS one, $7(12)$, e52249.

\section{Author Contributions}

472 TMS and KCR designed the experiments. TMS carried out the experiments. MGD and KCR

473 analyzed data. TMS, MGD, and KCR wrote the manuscript. 